\title{
$\beta$-Galactosidase and $\alpha$-L-Arabinofuranosidase Activities and Gene Expression in European and Chinese Pear Fruit During Ripening
}

\author{
Mercy W. Mwaniki',2, Francis M. Mathooko ${ }^{2}$,Kyoko Hiwasa ${ }^{3}$, Akira Tateishi ${ }^{4}$, \\ Naoki Yokotani ${ }^{1}$, Koichiro Ushijima ${ }^{1}$, Ryohei Nakano', Akitsugu Inaba' \\ and Yasutaka Kubo ${ }^{1 *}$
}

${ }^{1}$ Graduate School of Natural Science and Technology, Okayama University, Tsushima, Okayama 700-8530, Japan

${ }^{2}$ Department of Food Science and Technology, Faculty of Agriculture, Jomo Kenyatta University of Agriculture and Technology, P.O. Box 62000, Nairobi 00200, Kenya

${ }^{3}$ Department of Food Systems, Faculty of Food Culture, Kurashiki Sakuyo University, Nagao Tamashima, Kurashiki 710-0292, Japan

${ }^{4}$ College of Bioresource Sciences, Nihon University, Fujisawa, Kanagawa 252-8510, Japan

\begin{abstract}
Substantial decreases in cell wall bound galactosyl and arabinosyl residues are two of the most evident cell wall compositional changes that occur during fruit ripening. The roles of $\beta$-galactosidase ( $\beta$-Gal) and $\alpha$-L-arabinofuranosidase $(\alpha-\mathrm{Af})$, the enzymes responsible for these respective losses, were investigated and compared in European (Pyrus communis L. 'La France') and Chinese (Pyrus bretschneideri Rehd. 'Yali') pear fruits which exhibit different softening characteristics during ripening. The increase in the activities of $\beta$-Gal and $\alpha$-Af during ripening in both types of pear fruit correlated well with an increase in climacteric ethylene production, and a concomitant decrease in flesh firmness in 'La France' fruit. However, there was no noticeable decrease in 'Yali' flesh firmness even after 28 days of storage at room temperature. In both fruit types, enzyme activity and the accumulation of transcripts hybridizing with PpGAL1, PpGAL4, PpARF2, and PcARF1 increased with fruit ripening. Increases in gene expression and enzyme activities in 'Yali' fruit with no detectable softening during ripening indicate that $\beta$-Gal and $\alpha$-Af may not mediate difference in fruit softening between two pears, but that they could play some role(s) in cell wall changes, perhaps in cooperation with other cell wall-modifying enzymes such as polygalacturonase.
\end{abstract}

Key Words: alpha-L-arabinofuranosidase, beta-galactosidase, enzyme activity, gene expression, pear fruit softening.

\section{Introduction}

Fruit softening is one of the biochemical processes that take place during ripening. It not only contributes to the eating quality of many fruits, but also dictates the postharvest handling procedures of these commodities. Although it is widely believed that the overall softening process results from a number of changes, cell wall modifications have been shown to be the major determinant of fruit softening (Fischer and Bennett, 1991).

Received; June 2, 2006. Accepted; September 4, 2006.

This work was supported in part by a Grant-in-Aid for Scientific Research (grant no. 09660029 to YK) from the Ministry of Education, Culture, Sports, Science and Technology (MEXT), Japan. Ms. M.W. Mwaniki and Dr. F.M. Mathooko are grateful to MEXT for a postgraduate scholarship and the Japan Society for the Promotion of Science for an Invitation Fellowship for Research in Japan, respectively.

* Corresponding author (E-mail: ykubo@ cc.okayama-u.ac.jp).
However, these modifications are complicated and considered to involve the coordinated and interdependent actions of a range of cell wall-modifying enzymes and proteins (Brummell and Harpster, 2001).

Among the various hydrolases, polygalacturonase (PG) is the most widely studied. It plays a significant role in polyuronide depolymerization and particularly in the hydrolysis and solubilization of the pectin fraction that is covalently bound to the wall (Dawson et al., 1992; Rose et al., 2003), although transgenic trials on tomato have shown that $P G$ is neither necessary nor sufficient for fruit softening (Giovannoni et al., 1989). In pears, on comparing softening characteristics among different fruit cultivars, Hiwasa et al. (2004) attributed the different softening behaviors to differences in endo-PG activity on the cleavage of galacturonide linkages, and differing expressions of $P G$ genes. A loss of neutral sugars of the pectic side chains has also been associated with pectin 
solubilization, and is thought to possibly contribute to pectin mobilization during ripening (Dawson et al., 1992; Nara et al., 2001; Redgwell et al., 1992). These neutral sugar components usually exist as side chains attached to rhamnosyl residues of the rhamnogalacturonan backbone, and their release during fruit ripening is assumed to increase the sensitivity of enzymatic degradation or accessibility of other glycan hydrolases (Tateishi et al., 2005a).

$\beta$-Galactosidase $\quad(\mathrm{EC} \quad 3.2 .1 .23 ; \quad \beta$-Gal) $\quad$ and $\alpha$-L-arabinofuranosidase (EC 3.2.1.55; $\alpha$-Af) are a family of hydrolases that reduce the levels of cell wall galactosyl and arabinosyl residues, respectively, and are thought to be responsible for the conspicuous reduction in the level of such residues in many ripening fruits including 'Bartlett' pear (Ahmed and Labavitch, 1980a, 1980b), Japanese pear (Tateishi et al., 2001, 2005b), apple (Yoshioka et al., 1995), and tomato (Smith and Gross, 2000; Sozzi et al., 2002a). More recently, $\alpha$-Af enzyme PpARF2 was purified from Japanese pear fruit and shown to possess bifunctional activity of $\alpha$-Af $/ \beta$-xylosidase as an enzymatic property (Tateishi et al., 2005a). In our previous study, we characterized the expression of different members of the $\beta$-Gal gene family in 'La France' pear fruit during growth and the possible role of ethylene regulation during postharvest ripening (Mwaniki et al., 2005). While changes in enzyme activity and gene expression correlated with fruit softening, the divergent members of the $\beta$-Gal multigene family showed differential regulation characteristics.

Generally, European pear 'La France' undergoes dramatic softening with ethylene production during softening resulting in a melting texture, whereas Chinese pear 'Yali' does not exhibit fruit softening and its flesh remains crispy even at late ripening stages (Hiwasa et al., 2004). The aim of this study was to gain more insight into the possible roles of $\beta$-Gal and $\alpha$-Af in fruit softening. To this end, a new gene of pear $\alpha-A f, P c A R F 1$, was cloned from the European pear, and its specific expression pattern along that of $P p A R F 2$ and the Japanese pear $\beta$-Gal genes $P p G a l 1-6$ were characterized using gene-specific probes. We compared corresponding enzyme activities and gene expression patterns in 'La France' and 'Yali', two pear fruit types which exhibit different softening behaviours during ripening.

\section{Materials and Methods}

\section{Plant material and treatments}

European pear ( $P$. communis L. 'La France') and Chinese pear ( $P$. bretschneideri Rehd. 'Yali') fruits were harvested at the commercial maturity stage from orchards in Yamagata and Okayama Prefectures, respectively. They were treated with $5000 \mu \mathrm{L} \cdot \mathrm{L}^{-1}$ propylene, an analogue of ethylene, at $20^{\circ} \mathrm{C}$ for 7 days until sufficient autocatalytic ethylene production was detected; this was to synchronize the onset of ripening after which the fruit were held in ambient conditions at $20^{\circ} \mathrm{C}$. The ethylene production rate and fruit firmness were then determined as previously described (Hiwasa et al., 2003) initially and at appropriate intervals. Samples were frozen in liquid nitrogen and stored at $-80^{\circ} \mathrm{C}$ for subsequent analysis.

\section{$\beta$-Galactosidase and $\alpha$-L-arabinofuranosidase extrac- tion and assay}

Extraction of $\beta$-Gal and $\alpha$-Af was done according to the method described by Sozzi et al. (1998) with slight modifications. Two grams of frozen fruit samples was ground to a fine powder with a pestle and mortar in the presence of liquid nitrogen and transferred to tubes containing three volumes of cold $0.1 \mathrm{M}$ sodium acetate/ acetic acid buffer ( $\mathrm{pH} 5.0$ ) containing $1.4 \mathrm{M} \mathrm{NaCl}, 1 \mathrm{mM}$ $\mathrm{ZnCl}_{2}, 5 \mathrm{mM}$ 2-mercaptoethanol, and $1.5 \%(\mathrm{w} / \mathrm{v})$ polyvinylpolypyrrolidone. After shaking for $30 \mathrm{~min}$ at $4^{\circ} \mathrm{C}$ and centrifugation at $12000 \times \mathrm{g}$ for $20 \mathrm{~min}$, the supernatant was passed through a $0.45 \mu \mathrm{m}$ membrane filter and loaded onto PD-10 columns (GE Healthcare Life Science, NJ, USA) previously equilibrated with $0.1 \mathrm{M}$ sodium citrate/ citric acid buffer ( $\mathrm{pH}$ 4.5) for rapid desalting and buffer exchange. The columns were eluted with the same buffer and the eluates represented the crude enzyme extracts. All preparations for enzyme extraction were performed at $0-4^{\circ} \mathrm{C} . \beta$-Gal and $\alpha$-Af activities were assayed using $p$-nitrophenyl- $\beta$-D-galactopyranoside and $p$-nitrophenyl$\alpha$-D-arabinofuranoside, respectively, as substrates. The reaction mixture containing $120 \mu \mathrm{L}$ of $0.1 \mathrm{M}$ sodium citrate/citric acid buffer (pH 4.5), $100 \mu \mathrm{L}$ of $0.1 \%$ bovine serum albumin, $30 \mu \mathrm{L}$ of enzyme extract and $100 \mu \mathrm{L}$ of $13 \mathrm{mM}$ of the respective substrate solution was incubated at $37^{\circ} \mathrm{C}$ for $1 \mathrm{~h}$. The reaction was stopped by adding $250 \mu \mathrm{L}$ of $0.3 \mathrm{M}$ sodium carbonate, and the $p$-nitrophenol released was measured spectrophotometrically at $400 \mathrm{~nm}$. Enzyme activity was expressed per gram fresh weight.

\section{Cloning of cDNA encoding $\alpha$-Af}

RNA derived from ripening 'La France' fruit was used to obtain a cDNA fragment encoding $\alpha$-Af by RT-PCRbased cloning using degenerate primers designed based on the amino acid sequences of other $\alpha$-Afs in the database. For the isolation of full-length cDNA, a RACEPCR cloning method was performed using a Marathon cDNA amplification kit (Clontech, Palo Alto, CA, USA) according to the manufacturer's protocol. The genespecific primers used to amplify the $5^{\prime}$ end were $5^{\prime}$ TTCTTCGAAGAGATAAACCACGC-3' and 5'-TGCAACCCATCTGAGCCTGTCAGTG-3', while primers used for 3'-RACE were 5'-TGGCTCATTGCCAATATGGGTGTTC-3' and 5'-GTGTCTTACCATTTGTACAGGAAGC-3'. PCR was performed using high-fidelity DNA polymerase (Pfx DNA polymerase; Invitrogen, USA), cloned into pGEM-T Easy vector (Promega, USA), and sequenced using a DNA sequencer (model DSQ-1000, Shimadzu, Kyoto, Japan). The cDNA clone was designated PCARF1. 
$R N A$ extraction and gel-blot analysis

Total RNA was extracted using the hot borate method of Wan and Wilkins (1994), and gel blot analysis was performed. The probes for $\beta$-Gal and $\alpha$-Af were from cDNAs corresponding to full length sequences of Japanese pear $\beta$-Gal and $\alpha$-Af genes designated $P p G A L 1$, PpGAL2, PpGAL3, PpGAL4, PpGAL5, PpGAL6, and PpARF2, whose accession nos. are AB046543, AB190363, AB190364, AB190365, AB190366, AB190367, and AB195230, respectively (Tateishi et al., 2005a, 2005b). In addition, a PCARF1 probe obtained from full length cDNA corresponding to the European pear $\alpha$-Af gene which was cloned as mentioned above was also used. The probes used were prepared with the DIG probe synthesis kit (Roche Diagnostics, Gmbh, Mannhein, Germany) according to the manufacturer's instructions. After hybridization, the membranes were washed twice at $37^{\circ} \mathrm{C}$ with $2 \times \mathrm{SSC}$ solution containing $0.1 \%$ SDS for $15 \mathrm{~min}$ each and then twice at $68^{\circ} \mathrm{C}$ with $0.1 \times$ SSC solution containing $0.1 \%$ SDS for 30 min each. The signals were detected by chemiluminescence using CDP-Star (Roche Diagnostics).

\section{Results and Discussion}

\section{Cloning of cDNA encoding PcARF1}

One full-length cDNA was obtained from European pear fruit by RT-PCR and RACE-PCR-based cloning and designated PCARF1. Its deduced amino acid sequence shared over $96 \%$ identity with those of Japanese pear PpARF1 (AB073311) and apple MdAFasel (AY309436), but very low homology with another Japanese pear $\alpha$-Af, PpARF2 (AB195230), which was also used in this study. Phylogenic analysis (not shown) of selected $\alpha$-AFs genes grouped PCARF1 in Glycoside Hydrolase (GH) family 51 and $P p A R F 2$ in GH family 3. Indeed, GH family 51 has most of the $\alpha$-Af genes such as from the fruit of Japanese pear, apple, and tomato, and Arabidopsis. Based on substrate specificity, $P p A R F 2$ was shown to possess $\alpha$-Af as well as $\beta$-xylosidase activity (Tateishi et al., 2005a).
Characteristics offlesh firmness and ethylene production in 'La France' and 'Yali' fruits during ripening

In both 'La France' and 'Yali' fruits, no differences in ripening behavior between the control and propylenetreated fruit were detected (data not shown). Therefore, only propylene-treated fruits were used for the subsequent experiments.

As reported previously in 'La France' fruit (Hiwasa et al., 2003), ethylene production increased with the onset of ripening in a climacteric-like manner concomitant with a decrease in flesh firmness (Fig. 1A). On the other hand, 'Yali' fruit showed a massive production of ethylene (more than 15-fold that of 'La France' fruit) at the climacteric peak (Fig. 1B) but no significant difference in its flesh firmness throughout the experimental period. However, treatment of 'Yali' fruit with exogenous ethylene at the preclimacteric stage stimulated not only ethylene production but also the respiration rate, pigment and aroma development (data not shown), indicating that 'Yali' fruit is indeed responsive to ethylene, as previously reported (Hiwasa et al., 2004).

\section{Changes in $\beta$-Gal activity and gene expression during ripening}

$\beta$-Gal activity in both 'La France' and 'Yali' fruits was present, albeit at low levels before the onset of ripening (Fig. 2A, B). In 'La France', enzyme activity increased in a climacteric-like manner which was concomitant with the decrease in fruit firmness (Fig. 1A). However, there was no visible decline in enzyme activity in 'Yali' fruit even 28 days after harvest, although the increase in activity was more rapid during the early ripening period (Fig. 2B). $\beta-\mathrm{Gal}(\mathrm{s})$ has been isolated from various fruits including tomatoes (Smith and Gross, 2000), avocadoes (Tateishi et al., 2002), and Japanese pears (Kitagawa et al., 1995; Tateishi et al., 2001) and characterized in relation to the removal of galactosyl residues during fruit softening. Similar to observations made in 'La France' pear, increases in $\beta$-Gal activity with postharvest ripening
(A) 'La France'

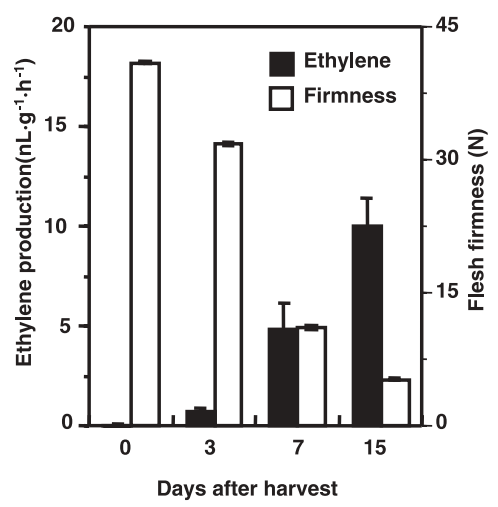

(B) 'Yali'

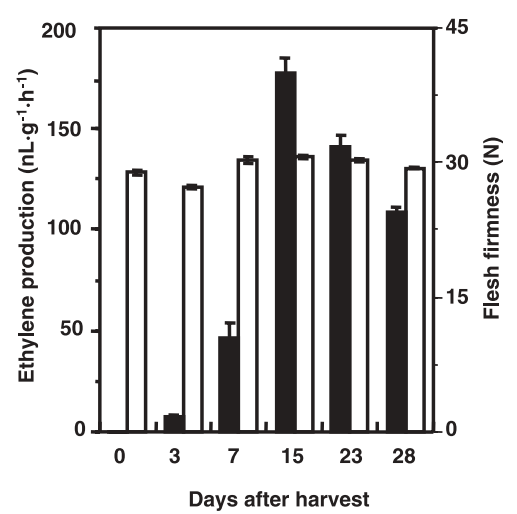

Fig. 1. Changes in ethylene production and flesh firmness in 'La France' (A) and 'Yali' (B) pear fruits during ripening. Fruits were harvested at commercial maturity and ripened at $20^{\circ} \mathrm{C}$. Each point represents the mean value of three replications and the vertical bars indicate $\pm \mathrm{SE}$ of the mean. 
(A) 'La France'

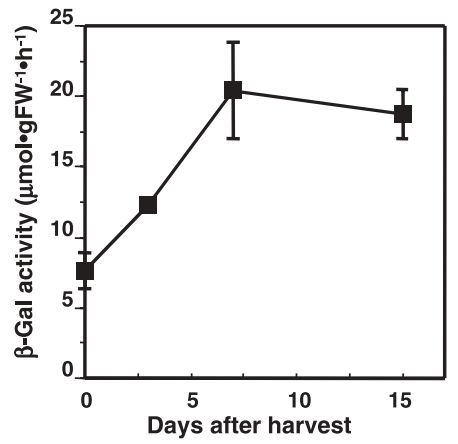

(C)

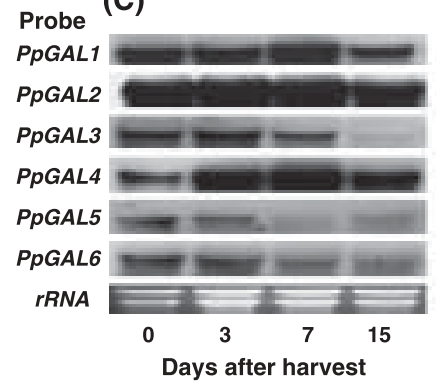

(B) 'Yali'

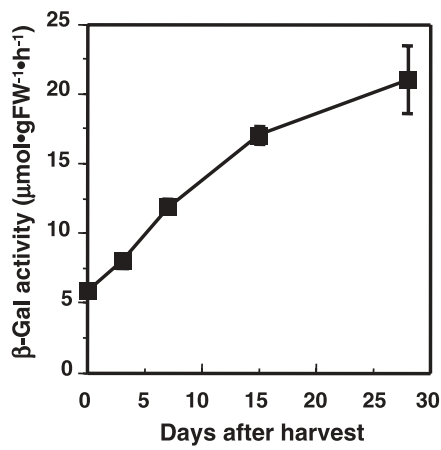

(D)

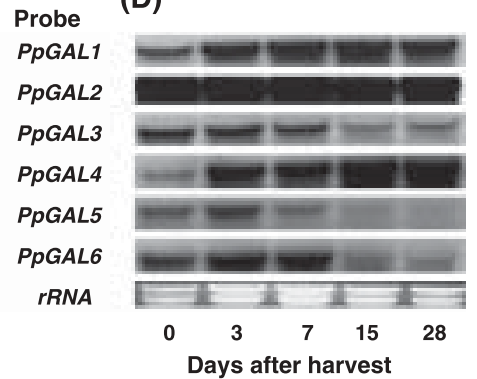

Fig. 2. Changes in $\beta$-galactosidase activity and accumulation of transcripts hybridizing with PpGAL1-6 probes in 'La France' (A) and 'Yali' (B) pear fruits during ripening. Conditions of fruit as indicated in Figure 1. Vertical lines on the bars represent $\pm \mathrm{SE}$ of three replications. Five micrograms of total RNA was used for RNA gel-blot analysis. Blots were hybridized using probes indicated on the left. Ethidium bromide staining of the gel is shown below the blots as a loading control.

and a decrease when there is little change in firmness has also been reported for 'Bartlett' pear (Ahmed and Labavitch, 1980b). Indeed, degalactosidation in response to $\beta$-Gal action has been proposed as an important process in cell wall modification during fruit ripening (Smith and Gross, 2000).

Similar to 'La France' and 'Yali' fruit, transcripts hybridizing with $P p G A L 1$ and $P p G A L 4$ were at high levels during ripening, $P p G A L 2$ transcripts were constitutively expressed throughout fruit ripening, while those of PpGAL3, PpGAL5, and PpGAL6 were limited rather to pre-ripening and early ripening stages (Fig. $2 \mathrm{C}, \mathrm{D}$ ). The expression of some genes was stimulated while, for others, it was suppressed upon the onset of ripening when ethylene production was induced. This is in agreement with our previous report (Mwaniki et al., 2005), and confirms that the differential expression of the different $\beta$-Gal genes in the ripening pear fruit could be due to down-and/or up-regulation by ethylene. The increases of enzyme activity in both 'La France' and 'Yali' fruit during ripening could be attributed to the increased accumulation of PpGAL1 and PpGAL4 transcripts. Tomato $\beta$-Gal $(T B G)$ gene members show similar unique patterns of expression during fruit growth and ripening, depicting their different developmental roles during tomato fruit ontogeny (Smith and Gross, 2000). For example, transgenic tomato fruit with suppressed ripening-related $T B G 4$ were found to be $40 \%$ firmer than controls during ripening, suggesting its possible involvement in cell wall modification (Smith et al., 2002). In our recent studies on the characterization of $\beta$-Gal family members in pears, we observed that the expression of $P p G A L 1$ and $P p G A L 4$ transcripts was not only fruit-specific but also ripeningspecific. Indeed phylogenic analysis of selected $\beta$-Gals showed TBG4, PpGAL1, and PpGAL4 to be closely related and grouped them in the same cluster (Mwaniki et al., 2005, Tateishi et al., 2005b). This study has further confirmed our earlier proposal that $P p G A L 1$ and $P p G A L 4$ might be of particular interest with respect to understanding the role they play in pear fruit softening.

\section{Changes in $\alpha$-Af activity and gene expression during ripening}

$\alpha$-Af activity and the accumulation of transcripts hybridizing with PpARF2 and PCARF1 probes were determined during off-tree ripening of two pear fruits types. In both fruits, $\alpha$-Af activity was undetectable at pre-ripening stages (Fig. 3A). In 'La France' fruit, an increase in enzyme activity was evident from the 3rd day of ripening after harvest but 7 th day in 'Yali' fruit. At the onset and progression of ripening, enzyme activity increased, at first sharply in the early ripening period and then gradually in the later ripening period. Increases in $\alpha$-Af activity in 'La France' fruit coincided with a decrease in flesh firmness, while no significant changes in 'Yali' fruit firmness (Fig. 1A, B) were observed throughout ripening despite it having a higher enzyme activity compared to 'La France' fruit. The possible 
(A)

'La France’

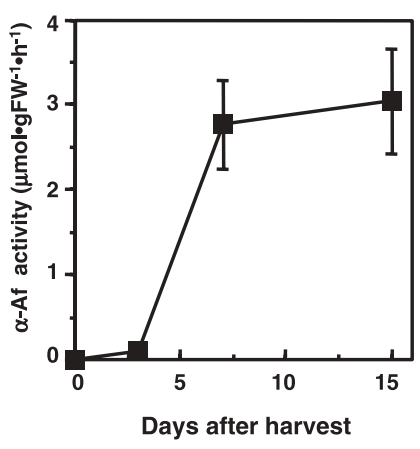

'Yali'

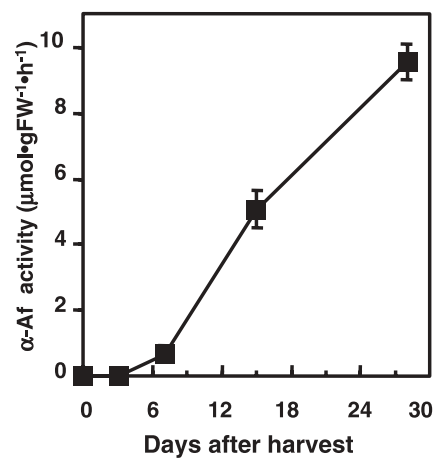

(B)

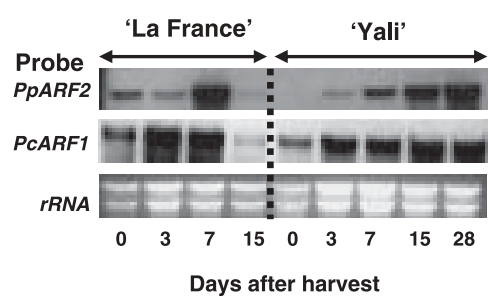

Fig. 3. Changes in $\alpha$-L-arabinofuranosidase activity (A) and accumulation of transcripts (B) hybridizing with $P p A R F 2$ and $P C A R F 1$ probes in 'La France' and 'Yali' pear fruits during ripening. Fruit treatment and conditions are as in Fig. 1. Five micro-grams of total RNA was used for RNA gel-blot analysis. Blots were hybridized using probes indicated on the left. Ethidium bromide staining of the gel is shown below the blots as a loading control.

involvement of glycosidases such as $\alpha$-Afs in cell wall modification during fruit development has been well documented. For example, Sozzi et al. (2002b) reported the presence of three $\alpha$-Af isoforms during tomato fruit development and ripening. They showed that one of the isoforms, designated $\alpha$-Af III, is specifically active during fruit softening and is probably associated with fruit softening. Further, expression of another tomato $\alpha$-Af gene, designated $L e A R F 1$, was shown to be negatively regulated by ethylene (Itai et al., 2003). This shows that the $\alpha$-Af family comprises divergent members which are responsive to different endogenous controls during fruit growth and development.

Transcripts hybridizing with $P p A R F 2$ and $P C A R F 1$ probes were differentially detected in the two fruit types. Accumulation of transcripts hybridizing with both $\alpha$-Afs was detected at low levels during the pre-ripening stage in both fruit types, and increased with ripening. However, in 'La France' fruit expression of both transcripts disappeared in the late ripening stage although there was some enzyme activity in this stage (Fig. 3A, B). This could be due to the existence of another $\alpha-A f$ gene(s) not identified by this study, responsible for the persistence of enzyme activity, or still, some activity of $\alpha$-Af persists long after its transcription and translation ceases.

The trend of enzyme activity during ripening in both pear types was more correlated with the accumulation of $P p A F R 2$ transcripts. This concurs with previous findings that showed the expression of $P p A R F 2$ to be ripeningrelated in the Japanese pear (Tateishi et al., 2005a). The observed lag between enzymatic activity and transcript accumulation of PCARF1 suggests a possibility that its activity could not be detected by the method of using the artificial substrate $p$-nitrophenyl- $\alpha$-D-arabinofuranoside, and the measurable $\alpha$-Af activity may have been a result of $P p A R F 2$. Another possibility, as mentioned above, is the existence of other $\alpha$-Afs members contributing to the enzyme activity and/or due to post transcriptional regulation of PCARF1.

In conclusion, it has been suggested that during fruit ripening, cell wall modification perhaps involves glycosidases such as $\beta-G a l$ and $\alpha$-Af acting in cooperation with pectolytic enzymes such as $\mathrm{PG}$ in pectin metabolism (Giovannoni, 2001). We previously observed that 'Yali' fruit lacked expression of the $P G$ gene which was abundant in 'La France' during fruit ripening (Hiwasa et al., 2004). It is therefore plausible that $\beta$-Gals and $\alpha$-Afs more likely contribute to cell wall modification in ripening fruit in cooperation with $\mathrm{PG}$ in pear. The increase of $\beta$-Gal and $\alpha$-Af enzyme activities and expression of their corresponding genes during ripening suggest that they somehow play a role in cell wall modification associated with fruit ripening in both European and Chinese pears. The results shown herein suggest that $\beta$-Gal and $\alpha$-Af may be necessary for pear fruit softening, and the activity and expression levels observed in 'Yali' may not have been sufficient to induce softening in this fruit. Therefore, the parameters analyzed in this study may not solely mediate pear fruit softening, and are therefore not adequate to explain the softening differences in the 
two pear fruit types. Further analysis will help to identify additional enzymes or factors contributing to pear fruit softening during ripening.

\section{Literature Cited}

Ahmed, A. E. and J. M. Labavitch. 1980a. Cell wall metabolism in ripening fruit I. Cell wall changes in ripening 'Bartlett' pears. Plant Physiol. 65: 1009-1013.

Ahmed, A. E. R. and J. M. Labavitch. 1980b. Cell wall metabolism in ripening fruit II. Changes in carbohydrate-degrading enzymes in ripening 'Bartlett' pears. Plant Physiol. 65: 1014 1016.

Brummell, D. A. and M. H. Harpster. 2001. Cell wall metabolism in fruit softening and quality and its manipulation in transgenic plants. Plant Mol. Biol. 47: 311-340.

Dawson, D. M., L. D. Melton and C. B. Watkins. 1992. Cell wall changes in nectarines (Prunus persica). Solubilization and depolymerization of pectic and neutral polymers during ripening and in mealy fruit. Plant Physiol. 100: 1203-1210.

Fischer, R. L. and A. B. Bennett. 1991. Role of cell wall hydrolases in fruit ripening. Ann. Rev. Plant Physiol. Plant Mol. Biol. 42: 675-703.

Giovannoni, J., D. DellaPenna, A. B. Bennett and R. L. Fischer. 1989. Expression of a chimeric polygacturonase gene in transgenic rin (ripening inhibitor) tomato fruit results in polyuronide degradation but not fruit softening. Plant Cell 1: 53-63.

Giovannoni, J. 2001. Molecular biology of fruit maturation and ripening. Ann. Rev. Plant Physiol. Plant Mol. Biol. 52: 725 749.

Hiwasa, K., Y. Kinugasa, S. Amano, R. Nakano, A. Inaba and Y. Kubo. 2003. Ethylene is required for both the initiation and progress of softening in pear (Pyrus communis L.) fruit. J. Exp. Bot. 54: 771-779.

Hiwasa, K., R. Nakano, A. Hashimoto, M. Matsuzaki, H. Murayama, A. Inaba and Y. Kubo. 2004. European, Chinese and Japanese pear fruits exhibit differential softening characteristics during ripening. J. Exp. Bot. 55: 2281-2290.

Itai, A., K. Ishihara and J. D. Bewley. 2003. Characterization of expression, and cloning, of $\beta$-D-xylosidase and $\alpha$-L-arabinofuranosidase in developing and ripening tomato (Lycopersicon esculentum Mill.) fruit. J. Exp. Bot. 54: 2615-2622.

Kitagawa, Y., Y. Kanayama and S. Yamaki. 1995. Isolation of $\beta$-galactosidase fractions from Japanese pear activity against native cell wall polysaccharides. Physiol. Plant. 93: 545-550.

Mwaniki, M. W., F. M. Mathooko, M. Matsuzaki, K. Hiwasa, A. Tateishi, K. Ushijima, A. Inaba and Y. Kubo. 2005. Expression characteristics of seven members of the $\beta$-galactosidase gene family in 'La France' pear (Pyrus communis L.) fruit during growth and their regulation by 1-methylcyclopropene during postharvest ripening. Postharvest Biol. Technol. 36: 253-263.
Nara, K., Y. Kato and Y. Motomura. 2001. Involvement of terminalarabinose and-galactose pectic compounds in mealiness of apple fruit during storage. Postharvest Biol. Technol. 22: 141150.

Redgwell, R. J., L. J. Melton and D. J. Brasch. 1992. Cell wall dissolution in ripening kiwifruit (Actinidia deliciosa). Solubilization of the pectic polymers. Plant Physiol. 98: 71-81.

Rose, J. K. C., C. Catalá, Z. H. Gonzalez-Carranza and J. A. Roberts. 2003. Cell wall disassembly. Ann. Plant Rev. 8: 264-324.

Smith, D. L. and K. C. Gross. 2000. A family of at least seven $\beta$-galactosidase genes is expressed during tomato fruit development. Plant Physiol. 123: 1173-1183.

Smith, D. L., J. A. Abbott and K. C. Gross. 2002. Down-regulation of tomato $\beta$-galactosidase 4 results in decreased fruit softening. Plant Physiol. 129: 1755-1762.

Sozzi, G. O., S. A. Camperi, O. Cascone and A. A. Fraschina. 1998. Galactosidases in tomato fruit ontogeny: decreased galactosidase activities in antisense ACC synthase fruit during ripening and reversal with exogenous ethylene. Aust. J. Plant Physiol. 25: $237-244$.

Sozzi, G. O., A. Fraschna, A. Navarro, O. Cascone, L. C. Greve and J. M. Labavitch. 2002a. $\alpha$-L-arabinofuranosidase activity during development and ripening of normal and ACC synthase antisense tomato fruit. HortScience 37: 564-566.

Sozzi, G. O., L. C. Greve, G. A. Prody and J. M. Labavitch. 2002 b. Gibberellic acid, synthetic auxins, and ethylene differentially modulate $\alpha$-L-arabinofuranosidase activities in antisense 1-aminocyclopropane-1-carboxylic acid synthase tomato pericarp discs. Plant Physiol. 129: 1330-1340.

Tateishi, A., H. Inoue, H. Shiba and S. Yamaki. 2001. Molecular cloning of $\beta$-galactosidase from Japanese pear (Pyrus pyrifolia $)$ and its gene expression with fruit ripening. Plant Cell Physiol. 42: 492-498.

Tateishi, A., H. Inoue and S. Yamaki. 2002. Cloning and expression of $\beta$-galactosidase cDNA related to softening of avocado (Persea americana) fruit. J. Japan. Soc. Hort. Sci. 71: 48-55.

Tateishi, A., H. Mori, J. Watari, K. Nagashima, S. Yamaki and H. Inoue. 2005a. Isolation, characterization and cloning of $\alpha$-L-arabinofuranosidase expressed during fruit ripening of Japanese pear. Plant Physiol. 138: 1653-1664.

Tateishi, A., K. Nagashima, F. M. Mathooko, M. W. Mwaniki, Y. Kubo, A. Inaba, S. Yamaki and H. Inoue. 2005b. Differential expression of members of the $\beta$-galactosidase gene family during Japanese pear (Pyrus pyrifolia L.) fruit growth and ontree ripening. J. Amer. Soc. Hort. Sci. 130: 819-829.

Wan, C. Y. and T. A. Wilkins. 1994. A modified hot borate method significantly enhances the yield of high-quality RNA from cotton (Gossypium hirsutum L.). Anal. Biochem. 223: 7-12.

Yoshioka, H., Y. Kashimura and K. Kaneko. 1995. $\beta$-D-Galactosidase and $\alpha$-L-arabinofuranosidase activities during the softening of apples. J. Japan. Soc. Hort. Sci. 63: 871-878. 